\title{
Application Optimal Control for a Problem Aircraft Flight
}

\author{
L. Kahina ${ }^{1,4, *}$, P. Spiteri ${ }^{2}$, F. Demim ${ }^{3}$, A. Mohamed ${ }^{4}$, A. Nemra ${ }^{3}$ and F. Messine ${ }^{5}$
}

${ }^{1}$ Université de Bouira. Faculté des Sciences et Sciences appliquées. Département de Mathématiques. Algérie. ${ }^{2}$ ENSEEIHT-IRIT, Université de Toulouse, France.

${ }^{3}$ Laboratoire Robotique et Productique, Ecole Militaire Polytechnique, Bordj El Bahri, Alger. Algérie.

${ }^{4}$ Laboratoire de Conception et Conduite de Systèmes de Production (L2CSP) Tizi-Ouzou, Algeria. ${ }^{5}$ ENSEEIHT-Laplace, Université de Toulouse, France.

Received 9 October 2017; Accepted 17 February 2018

\begin{abstract}
Our aim is to solve a problem of optimal control with free final time using the Pontryagin's maximum principle. As an illustration, we consider a navigation problem which is solved analytically and numerically by the shooting method in the case without constraint. The two approaches are compared. In the second case, we solve numerically the same problem with constraint on the state. At the end, we prove the convergence of the method for the second case.
\end{abstract}

Keywords: Optimal control, Shooting method, Pontryaguin principle's.

\section{Introduction}

Indirect methods based on the principle of maximum Pontryaguin $[1,2,4]$ are known for their speed and precision in the treatment of optimal control problems. In the present study, we consider an optimal control problem with free final time. In order to illustrate this study, we consider a problem of aircraft flying from an initial state to a final state. We are particularly interested in minimizing the duration landing pass from an initial position to a final one. For this, we will use the principle of Pontryaguin [4, 15, 16, 17], and determine the optimality equations resulting from this principle; i.e.; a differential-algebraic system as the state equation is provided (with an initial condition and a final condition) and the adjoint equation. On other hand, note that in the adjoint equation, derived from the principle's Pontryaguin, no information is given concerning the initial or the final conditions; consequently this costate equation is hard to use algorithmically[12, 13, 14, 17]. Thus, in order to determine the initial condition of the adjoint state, we use the shooting indirect method for the numerical procedure [4]. Note also, that we consider in the presented study two distinct cases corresponding to the cases where constraints are submitted or not submitted to the state. Finally, we present the results of numerical experiments implemented using Matlab facilities.

\section{Statement of problem}

The optimal control problem considered is to find the control $u(t)$ that minimizes the performance index

*E-mail address: louadi_kahina@yahoo.fr

ISSN: 1791-2377 @ 2018 Eastern Macedonia and Thrace Institute of Technology. All rights reserved. doi:10.25103/jestr.111.19
$J=\varphi\left(t_{f}, x_{f}\right)+\int_{t_{0}}^{t} L(t, x, u) d t$

subject to the differential constraints

$\dot{x}=f(t, x, u)$;

the prescribed initial condition at the initial time $t_{0}$

$x\left(t_{0}\right)=x_{0}$,

and prescribed final conditions at the final time $t_{f}$

$\psi\left(x_{f}, t_{f}\right)=0$,

here, $\psi$ is a $(l+1) \times 1-$ vector, where $0 \leq l \leq n$; there must be at least one final condition that draws the optimal path to the final value.

In order to use the Pontryaguin principle's, the Hamiltonian $H$ is defined by:

$H=L(t, x, u)+p^{T} f(t, x, u)$

The Euler-Lagrange equations are given by:

$\dot{x}=f(t, x, u)$,

$\dot{p}=-H_{x}^{T}(t, x, u, p)$,

$0=H_{u}^{T}(t, x, u, p)$, 
and the previous prescribed boundary conditions

$$
x\left(t_{0}\right)=x_{0}
$$

$\psi\left(t_{f}, x_{f}\right)=0$.

We will find $u^{*}$ which minimizes $H$ such that $u^{*}$ verify the following inequality

$$
H\left(t, x, u^{*}, p\right)-H(t, x, u, p) \leq 0, \forall u
$$

\subsection{Transversality condition on $p$}

Generally, when the terminal cost is considered in the cost functional, the functional to be minimized can be written as follows :

$$
J=\varphi\left(t_{f}, x_{f}\right)+\int_{t_{0}}^{t} L(t, x, u) d t
$$

Let $M_{0}$ and $M_{1}$ be two subsets of $\mathbf{R}^{n}$; then to minimize the cost functional one should find a trajectory between $M_{0}$ and $M_{1}$. Moreover if $M_{0}$ and $M_{1}$ are two varieties of $\mathbf{R}^{n}$ having the tangent spaces $T_{x_{0}} M_{0}$ and $T_{x\left(t_{f}\right)} M_{1}$ respectively $x_{0} \in M_{0}$ and $x_{t_{f}} \in M_{1}$, then the vector $p(t)$ must verify the transversality conditions:

$$
\begin{aligned}
& p(0) \perp T_{x_{0}} M_{0}, \\
& p\left(t_{f}\right)-p^{0} \nabla_{x} \varphi\left(t_{f}, x_{f}\right) \perp T_{x_{f}} M_{1},
\end{aligned}
$$

where $p^{0}$ is a real such that $p^{0}<0$ leads to the Pontryaguin's maximum principle and $p^{0}>0$ leads to the Pontryaguin's minimum principle [4]. If $M_{0}=x_{0}$, the condition (13) becomes empty and the variety $M_{1}$ can be written as follows:

$$
M_{1}=\left\{x \in \mathbf{R}^{n} / F_{1}(x)=F_{2}(x)=\ldots=F_{q}(x)=0\right\},
$$

where $F_{i}$ are functions of class $C^{1}$ on $\mathbf{R}^{n}$; then the tangent space to $M_{1}$ at a point $x \in M_{1}$ is defined by:

$$
T_{x} M_{1}=\left\{v \in \mathbf{R}^{n} / \nabla F_{i}(x) v=0, i=1, \ldots, q\right\}
$$

and the condition (14) is written as follows:

$$
\exists v_{1}, \ldots v_{q} \in \mathbf{R} / p\left(t_{f}\right)=\sum_{i=1}^{q} v_{i} \nabla_{x} F_{i}\left(x\left(t_{f}\right)\right)+p^{0} \nabla_{x} \varphi\left(t_{f}, x_{f}\right)
$$

where $v_{i}$ are the Lagrange multipliers.

The transversality condition of Hamiltonian is defined by:

$$
H\left(t_{f}, x_{f}, p\left(t_{f}\right), p_{0}, u\left(t_{f}\right)\right)=0 .
$$

corresponding to the fact that the Hamiltonian vanishes at final time.

\section{Shooting Indirect method}

The shooting indirect method is used to obtain the value of $p(0)$ necessary to the solution of the problem characterized by the Pontryaguin principle. If it is possible, from the condition of minimization of the Hamiltonian to express the control extremal function of $(x(t), p(t))$ then the extremal system is a differential system of the form $\dot{z}(t)=G(t, z(t))$ where $z(t)=(x(t), p(t))$. With a numerical integrator from $z_{0}$ we obtain : $\tilde{z}_{i}^{z} 0: z\left(t_{i}\right)$, where the $t_{i} \quad i=1,2, \ldots$ are the time moments discretized by the integrator. But in $z_{0}=\left(x_{0}, p_{0}\right)$, the value $x_{0}$ is given by the initial condition. Then, by doing some variations on $p_{0}$, we obtain the different $\tilde{z}_{i}^{z}{ }^{2}$. Which interests us are the $\tilde{z}_{N}^{z_{0}}: z\left(t_{f}\right)$ (at final time); else $\tilde{z}_{N}^{z}=\left(\tilde{x}_{N}^{z}, \tilde{p}_{N}^{z}\right)$ and only the $\tilde{x}_{N}^{z}$ are significant. Since they depend only on $p_{0}$, note that $\tilde{x}_{N}^{p_{0}}$. Let $G$ be the implicit function giving $p_{0}$ by numerical calculation using an integrator returns $\tilde{x}_{N}^{p_{0}}-x_{f}$ :

$G: \mathbf{R}^{n} \rightarrow \mathbf{R}^{n}$ and

$G\left(p_{0}\right)=\tilde{x}_{N}^{p_{0}}-x_{f}$.

Here $G$ is an implicit nonlinear system of $n$ equations and $n$ unknowns:

$G\left(p_{0}\right)=0$.

For the solution, we used the Newton's method. The principle of the Newton's method is described as follows: in the $k$-th step, let $p_{0}^{k}$ be an approximation of the zero $p_{0}$ of $G$; therefore $p_{0}$ can be written $p_{0}=p_{0}^{k}+\Delta p_{0}^{k}$, and then:

$0=G\left(p_{0}\right)=G\left(p_{0}^{k}+\Delta p_{0}^{k}\right)$

$=G\left(p_{0}^{k}\right)+\frac{\partial G}{\partial p_{0}}\left(p_{0}^{k}\right) \cdot\left(p_{0}-p_{0}^{k}\right)+o\left(p_{0}-p_{0}^{k}\right)$

which leads to the solution of

$\frac{\partial G}{\partial p_{0}}\left(p_{0}^{k}\right) \cdot\left(p_{0}-p_{0}^{k}\right)=-G\left(p_{0}^{k}\right)$ 
L. Kahina, P. Spiteri, F. DEMIM, A. Mohamed, A. Nemra and F. Messine/

Journal of Engineering Science and Technology Review 11 (1) (2018) 156-164

where $\frac{\partial G}{\partial p_{0}}\left(p_{0}^{k}\right)$ is the Jacobian matrix of the application $p_{0} \rightarrow G\left(p_{0}\right)$ computed when $p_{0}=p_{0}^{k} ;$ note that the mapping $p_{0} \rightarrow G\left(p_{0}\right)$ is not explicitly known but is known numerically. So we will use a method of numerical derivation based on the finite difference. To avoid the calculation of $\frac{\partial G}{\partial p_{0}}\left(p_{0}^{k}\right)$, it suffices to find an approximation of $\frac{\partial G}{\partial p_{0}}\left(p_{0}^{k}\right)$; according to [3], we will use one of the following finite difference approximations.

$\frac{\partial G_{i}}{\partial p_{0}}\left(p_{0}^{k}\right) \approx \frac{1}{h_{i j}}\left[G_{i}\left(p_{0}+\sum_{k=1}^{j} h_{i k} e^{k}\right)\right.$

$\left.-G_{i}\left(p_{0}+\sum_{k=1}^{j-1} h_{i k} e^{k}\right)\right]$

or else

$\frac{\partial G_{i}}{\partial p_{0}}\left(p_{0}^{k}\right) \approx \frac{1}{h_{i j}}\left[G_{i}\left(p_{0}+h_{i j} e^{j}\right)-G_{i}\left(p_{0}\right)\right]$

where the $h_{i j}$ are the given discretization step of the $i-t h$ equation with respect to the $j-t h$ variable, and $e^{k}$ are the $k$-th vector of the canonical basis; note that, classically, we can always choose the values of $h_{i j}$ equal each other at a common value $h$. Let $\Delta_{i j}\left(p_{0}, h\right)$ be a finite difference approximation, then we have:

$\lim _{h \rightarrow 0} \Delta_{i j}\left(p_{0}, h\right)=\frac{\partial G_{i}}{\partial p_{0 j}}\left(p_{0}\right), i, j=1, \ldots, n$.

Let,

$J\left(p_{0}, h\right)=\left(\Delta_{i j}\left(p_{0}, h\right)\right)$

which is an approximation of the Jacobian matrix; then the approximate Newton's method can be written as follows $p_{0}^{k+1}=p_{0}^{k}-J\left(p_{0}^{k}, h^{k}\right)^{-1} \cdot G\left(p_{0}^{k}\right)$.

The problem of convergence of this iterative process is ensured by using a result of the book of Ortega and Rheinboldt [6]; indeed if the discretization step $h_{i j}$ are small and tend to zero, the convergence is ensured.

\section{Navigation problem}

\subsection{Case without constraint on the state}

Consider the problem of flying an aircraft with a constant speed crosswind from one point to another in minimum time. Figure (1), describes the simplified form of Zermelo's problem [1]. Note that $t_{f}$ is free, as it must be to have a minimum time problem.

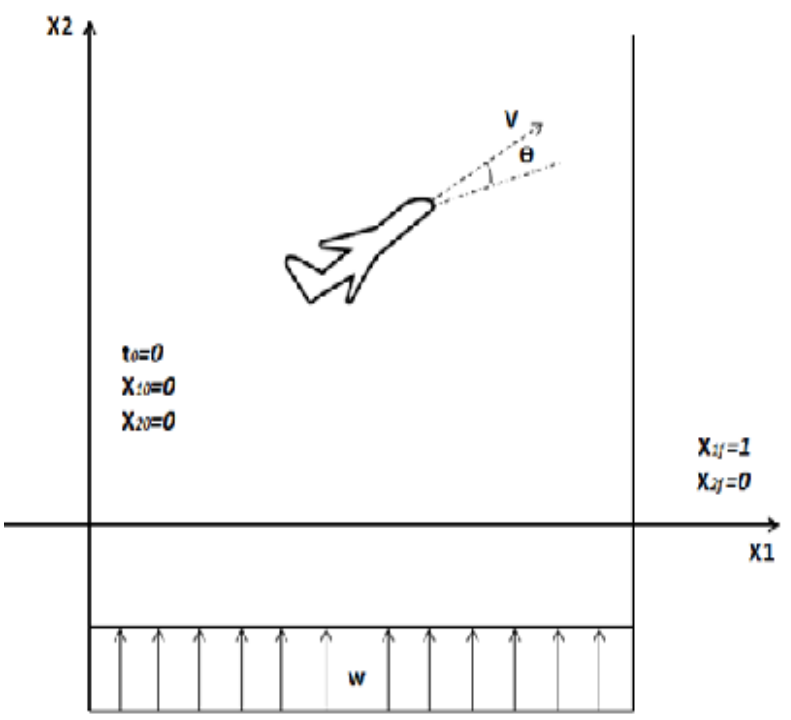

Fig. 1. Navigation problem

The optimal control problem is stated as follows: Find the control $\theta(t)$ that minimizes the final time

$J=t_{f} \rightarrow \min$,

subject to the differential constraints

$\dot{x}_{1}=V \cos \theta$

$\dot{x}_{2}=V \sin \theta+w$,

Where,

- $x_{1}$ et $x_{2}$ are the Cartesian coordinates,

- $V$ is the constant speed of the aircraft relative to the air,

- $\theta$ is the controllable orientation of aircraft velocity vector relative to the ground,

- $w$ is the speed of the air relative to the ground.

The prescribed boundary conditions:

$t_{0}=0, x_{10}=0, x_{20}=0$,

$x_{1 f}=1, x_{2 f}=0$.

The functional (15) is equivalent to:

$\int_{0}^{t} d t \rightarrow \min$

The Hamiltonian is given by: 
$H=p_{1}(V \cos \theta)+p_{2}(V \sin \theta+w)-1$.

The Euler-Lagrange equations leads to:

$\dot{x}_{1}=H_{p 1}=V \cos \theta$

$\dot{x}_{2}=H_{p 2}=V \sin \theta+w$,

$\dot{p}_{1}=-H_{x 1}=0$,

$\dot{p}_{2}=-H_{x 2}=0$,

$0=H_{\theta}=-p_{1} V \sin \theta+p_{2} V \cos \theta$,

From (58)-(59), it follows that:

$p_{1}=$ constante, $p_{2}=$ constante .

Then, the equation for $\theta$ gives:

$-p_{1} \sin \theta+p_{2} \cos \theta=0$.

Which, since $p_{1}$ and $p_{2}$ are constant, implies that $\operatorname{tg} \theta$ is constant and then $\theta$ is also constant.

From the equation (54), we deduce that:

$x_{1}=(V \cos \theta) t$,

From the equation (55), we obtain:

$x_{2}=(V \sin \theta+w) t$.

Using the prescribed final conditions $x_{1 f}=1$ and $x_{2 f}=0$, leads to:

$t_{f}=\frac{1}{V \cos \theta}, \sin \theta=-\frac{w}{V}$.

Then, from Figure (2), we obtain:

$\cos \theta=\frac{\sqrt{V^{2}-w^{2}}}{V}$,

Hence, the optimal control and the final time can be written as:

$\theta=-\arcsin (w / V), t_{f}=\frac{1}{V^{2}-w^{2}}$.

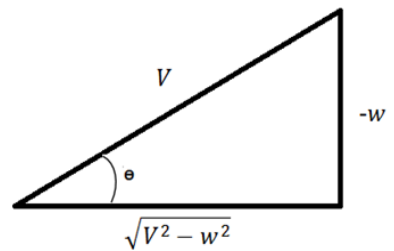

Fig. 2. Control Triangle
The boundary conditions for this problem are given by:

$H_{f}=-1, p_{1 f}=v_{1}, p_{2 f}=v_{2}$;

The value $p_{1}\left(v_{1}\right)$ is given by:

$p_{1}=-\frac{1}{\sqrt{V^{2}-w^{2}}}$.

From the equation (27), we obtain:

$p_{2}=\frac{w}{V^{2}-w^{2}}$.

Finally, we obtain the following results:

$\theta=-\arcsin (w / V), t_{0}=0, t_{f}=\left(V^{2}-w^{2}\right)^{-1 / 2}$

$x_{1}=\sqrt{V^{2}-w^{2}} t, x_{10}=0, x_{1 f}=1$

$x_{2}=(V \sin \theta+w) t, x_{20}=0, x_{2 f}=0$

$p_{1}=-\left(V^{2}-w^{2}\right)^{-1 / 2}=p_{10}=p_{1 f}$

$p_{2}=w /\left(V^{2}-w^{2}\right)=p_{20}=p_{2 f}$.

Note that the aircraft is pointing upwind but it is moving directly toward the final point. An interesting check of the results can be made for the case where $w<<V$. Here, $\theta \cong 0, x_{1} \cong V t$, and $x_{2} \cong 0$, which seem reasonable.

To test the minimality of the solution, application of the Weierstrass condition leads to

$p_{1} V \cos \theta_{*}+p_{2}\left(V \sin \theta_{*}+w\right)-$

$-p_{1} V \cos \theta-p_{2}(V \sin \theta+w)>0$.

Since $V \neq 0$, and cancelation of the term $p_{2} w$ gives:

$p_{1} \cos \theta_{*}-p_{2} \sin \theta_{*}-p_{1} \cos \theta-p_{2} \sin \theta>0$

Then, the optimality condition (27) becomes:

$p_{2}=p_{1} \tan \theta$

The application of the Legendre-Clebsch condition to this problem starts with :

$$
H_{\theta \theta}=-p_{1} V \cos \theta-p_{2} V \sin \theta
$$

If the values for $p_{1}, p_{2}$, and $\theta$ from the equation (32) are substituted into the expression of $H_{\theta \theta}$, we obtain: 


$$
H_{\theta \theta}=\frac{V^{2}}{V^{2}-w^{2}}>0 .
$$

Since $w<V, H_{\theta \theta}$ are positive.

\section{Numerical application}

For $V=300 \mathrm{~km} / \mathrm{h}, w=40 \mathrm{~km} / \mathrm{h}$, then

$\theta=-0.1337$ radians, $t_{0}=0, t_{f}=0.2040 m n$

$x_{1}=297.3214 t, x_{10}=0, x_{1 f}=1$

$x_{2}=-164.7839 t, x_{20}=0, x_{2 f}=0$

$p_{1}=-0.0034=p_{10}=p_{1 f}$

$p_{2}=4.5249 e-004=p_{20}=p_{2 f}$

For different values of $V$ and $w$, the analytical solution allows to obtain the following results:

Table 1. Analytical solution

\begin{tabular}{c|c|c|c}
\hline $\mathbf{V}(\mathbf{K m} / \mathbf{h})$ & $\mathbf{w}(\mathbf{K m} / \mathbf{h})$ & $\mathbf{t}_{\mathbf{f}}(\mathbf{m n})$ & $\boldsymbol{\theta}$ (radians) \\
\hline 300 & 40 & 0.2040 & -0.1337 \\
350 & 50 & 0.1740 & -0.1433 \\
400 & 60 & 0.1517 & -0.1506 \\
450 & 70 & 0.1350 & -0.1562 \\
500 & 80 & 0.1216 & -0.1607 \\
600 & 100 & 0.1014 & -0.1674 \\
700 & 120 & 0.0870 & -0.1723 \\
800 & 140 & 0.0762 & -0.1759 \\
900 & 170 & 0.0679 & -0.1900 \\
\hline
\end{tabular}

\section{Numerical solution}

For the numerical solution, we used the shooting indirect method. Then we have to solve the following system :

$\dot{z}_{1}=V \cos \theta$

$\dot{z}_{2}=V \sin \theta+w$,

$\dot{z}_{3}=0$,

$\dot{z}_{4}=0$,

$\theta=\operatorname{arctang} \frac{z_{4}}{z_{3}}$

$z_{1}(0) \in \mathbf{R}, z_{2}(0) \in \mathbf{R}$,

$z_{3}(0) \in \mathbf{R}, z_{4}(0) \in \mathbf{R}$.

Let $z(t)$ be the solution of the previous system at time $t$ with the initial conditions

$z(0)=\left(z_{1}(0), z_{2}(0), z_{3}(0), z_{4}(0)\right)$.

Let $z(0)=(x(0), p(0))$.

We construct a shooting function which is a nonlinear algebraic equation of the variable $p$ at time $t=0$. This shooting function is computed by a numerical procedure of integration of ordinary differential equation (using for example Euler method, Runge-Kutta method,. . . ); the shooting function is defined by:

$G(z(0))=\left(\begin{array}{c}z_{1}\left(t_{f}, 0,0, p_{1}, p_{2}\right)-1 \\ z_{2}\left(t_{f}, 0,0, p_{1}, p_{2}\right)\end{array}\right)$.

The problem to solve is then written: Find $p(0)$ such that $G(z(0))$ gives the desired value of $x\left(t_{f}\right)$. The algorithm for numerical solution of this problem will then be completely defined if one gives oneself:

1. the integration algorithm of a differential system with initial condition (e.g., Euler or Runge-Kutta procedure) to compute the shooting function $G$ (implemented in 'ode 45 ' of Matlab which is a method of Runge-Kutta $4 / 5$ with variable pitch).

2. the solution algorithm $G(z)=0$ which in our case uses the method quasi- newton (implemented in 'fsolve' of Matlab).

For different values of the $V$ and $w$, we obtain the following figures that shows the state and the control.
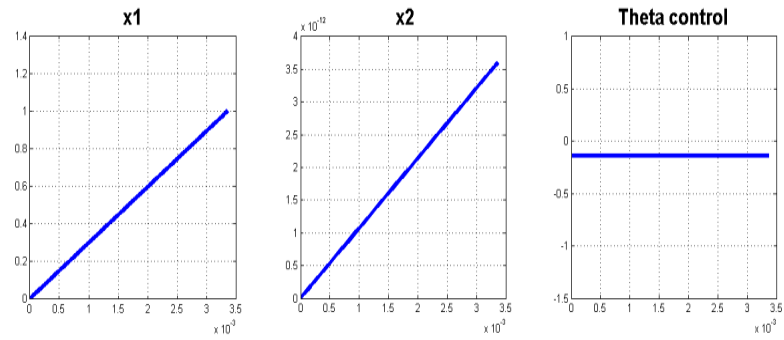

Fig. 3. State and control for a speed of aircraft $V=300$ and of wind $\mathrm{w}=40$
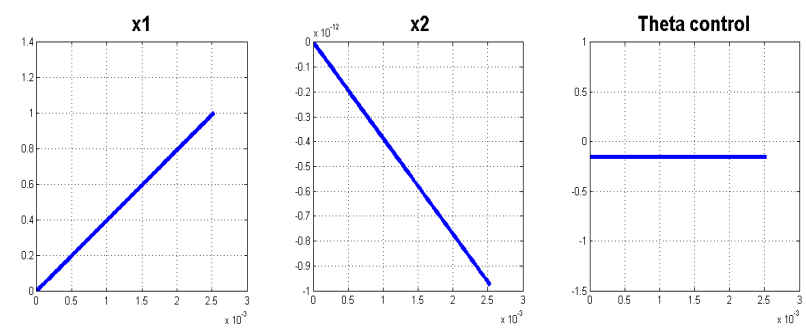

Fig. 4. State and control for a speed of aircraft $V=400$ and of wind $\mathrm{w}=60$
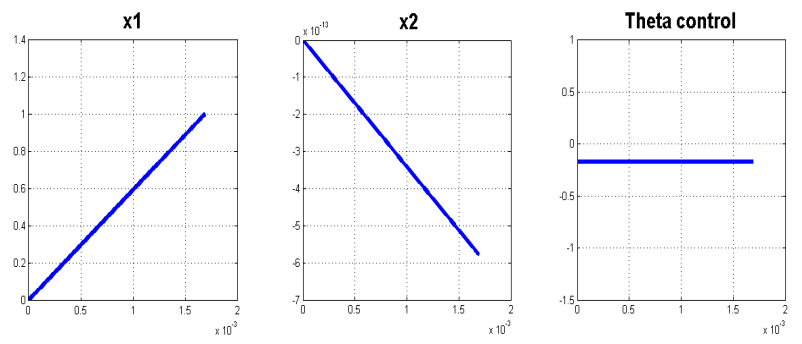

Fig. 5. State and control for a speed of aircraft $V=600$ and of wind $\mathrm{w}=100$ 

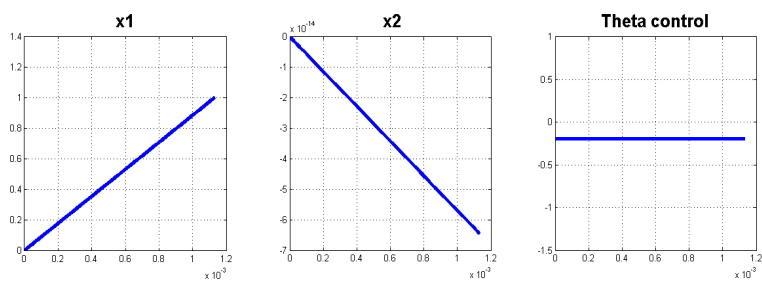

Fig. 6. State and control for a speed of aircraft $V=900$ and of wind $\mathrm{w}=170$

The results are in the Table 2:

Table 2:Numerical solution

\begin{tabular}{c|c|c|c|c|c}
\hline $\begin{array}{c}V \\
\mathbf{( k m} / \mathbf{h})\end{array}$ & $\begin{array}{c}w \\
\mathbf{( k m} / \mathbf{h})\end{array}$ & & $\theta$ & Itérations & $\begin{array}{c}\text { Time } \\
\text { (seconds) }\end{array}$ \\
\hline 300 & 40 & 0.1860 & -0.1980 & 6 & 0.19 \\
& & & & & \\
350 & 50 & 0.1680 & -0.1433 & 6 & 0.20 \\
400 & 60 & 0.1500 & -0.1506 & 6 & 0.22 \\
450 & 70 & 0.1320 & -0.1562 & 6 & 0.20 \\
500 & 80 & 0.1140 & -0.1607 & 6 & 0.21 \\
600 & 100 & 0.0960 & -0.1674 & 6 & 0.21 \\
700 & 120 & 0.0840 & -0.1723 & 6 & 0.21 \\
800 & 140 & 0.0763 & -0.1759 & 6 & 0.21 \\
900 & 170 & 0.0660 & -0.1900 & 6 & 0.20 \\
\hline
\end{tabular}

We deduce that the exact solution and the numerical solution are similar (see Tables 1 and 2). The performance of the numerical procedure are summarized in Table 2, for different values of $V$ and $w$. Note that the convergence is fast, moreover, the computation time is very low for a number of iterations not large enough. Note that when the speed of the wind increases, then, the time of landing decreases. In addition, it should be noted that wind is an important factor that reduces the duration of landing. In fact, during the flight, the aircraft must navigate in an air lane.

\subsection{Case with constraints on the state}

In this section, we consider a more complex situation. Then, a more realistic modelisation of the navigation of the aircraft will be better described by considering (15)-(56) in which $\underline{x}_{i} \leq x_{i}(t) \leq \bar{x}_{i}, i=1,2, \quad \underline{x}_{i}$ and $\quad \bar{x}_{i}$ being the extremal values of the state variables $x_{i}, i=1,2$. In such a case, the analytical procedure can not be applied, but the numerical procedure is well adapted. From a practical point of view, during the numerical procedure, we have to project the values of $x_{i}, i=1,2$, on the convex set describing the constraints in the state variables.

$$
J=\varphi\left(t_{f}, x_{f}\right)+\int_{t_{0}}^{t_{f}} L(t, x, u) d t
$$

subject to the differential constraints

$$
\dot{x}=f(t, x, u)
$$

the prescribed initial condition at the initial time $t_{0}$ $x\left(t_{0}\right)=x_{0}$,

and prescribed final conditions at the final time $t_{f}$

$\psi\left(x_{f}, t_{f}\right)=0$,

here, $\psi$ is a $(l+1) \times 1-$ vector, where $0 \leq l \leq n$; there must be at least one final condition that draws the optimal path to the final value.

We consider a constraint of the state $g(t, x, u) \geq 0$ To state the maximum principle, we define the Hamiltonian function as:

$H=L(t, x, u)+p^{T} f(t, x, u)$

we also define the Lagrangian function as

$\mathrm{L}(t, x, u, p, \mu)=H(t, x, p, u, p)+\mu g(t, x, u)$

where $\mu \in R$ is a row vector, whose components are called Lagrange multipliers. These Lagrange multipliers satisfy the following condition:

$\mu \geq 0,<\mu, g(t, x, u)>=0$

The adjoint vector satisfies the differential equation:

$\dot{p}=-L_{x}^{T}(t, x, u, p)$,

with boundary conditions

$p\left(t_{f}\right)=\varphi\left(t_{f}, x_{f}\right)+\alpha \psi_{x_{f}}\left(t_{f}, x_{f}\right)$

where $\alpha \geq 0,<\alpha, \psi_{x_{f}}\left(t_{f}, x_{f}\right)>=0$ with $\alpha$ is constant vector.

The maximum principles states that the necessary condition for $u^{*}$, with corresponding state trajectory $x^{*}$, to be an optimal control are there should exist continuous and piecewise continuously differentiable function $p$, piecewise continuous function $\mu$, and constant $\alpha$ such that the following conditions are verified:

$\dot{x}^{*}=f\left(t, x^{*}, u^{*}\right), x^{*}(0)=x_{0}$

satisfying the terminal constraint

$\psi\left(t_{f}, x_{f}\right)=0$

$\dot{p}=-L_{x}^{T}(t, x, u, p)$

with the transversality condition

$p\left(t_{f}\right)=\nabla_{x} \varphi\left(t_{f}, x_{f}\right)+\alpha \psi_{x_{f}}\left(t_{f}, x_{f}\right)$ 
$\alpha \geq 0,<\alpha, \psi_{x_{f}}\left(t_{f}, x_{f}\right)>=0$

the Hamiltonian maximizing condition

$H\left(t, x^{*}(t), u^{*}(t), p(t)\right) \geq H\left(t, x^{*}(t), u^{*}(t), p(t)\right)$

at each $t \in\left[0, t_{f}\right]$

for all $u$ satisfying $g(t, x, u) \geq 0$ and the Lagrange multipliers $\mu(t)$ are such that

$\left.\left.\frac{\partial L}{\partial u}\right|_{u=u} *\left(\frac{\partial H}{\partial u}+\mu \frac{\partial g}{\partial u}\right)\right|_{u=u} * 0$

and the complementary condition

$\mu(t) \geq 0,<\mu(t), g\left(t, x^{*}, u^{*}\right)>=0$ hold by:

The transversality condition of Hamiltonian is defined

$$
H\left(t_{f}, x_{f}, p\left(t_{f}\right), p_{0}, u\left(t_{f}\right)\right)+\varphi\left(t_{f}, x_{f}\right)=0
$$

\subsection{Sufficiency condition}

\section{Definitions}

- A function $f: D \rightarrow E$ is concave, if for all $x_{1}, x_{2} \in D$ and for all $\lambda \in[0,1]$,

$f\left(\lambda x_{1}+(1-\lambda) x_{2}\right) \geq \lambda f\left(x_{1}\right)+(1-\lambda) f\left(x_{2}\right)$,

this definition is equivalent to:

If $f^{\prime \prime}(x) \geq 0$ on $D$, then $f$ is concave on $D$.

- The function $f$ is quasiconcave if

$f\left(x_{1}\right) \leq f\left(x_{2}\right) \Rightarrow f\left(\lambda x_{1}+(1-\lambda) x_{2}\right) \geq f\left(x_{1}\right)$

Theorem 5.1 [5] Let $\left(t, x^{*}, u^{*}, p, \mu, \alpha\right)$ satisfy the necessary conditions in (52). If $H(t, x(t), u(t), p(t))$ is concave in $(x, u)$ at each $t \in\left[0, t_{f}\right], \varphi$ is concave in $x, g$ quasiconcave in $(x, u), \quad \psi$ is concave in $x$, then $\left(x^{*}, u^{*}\right)$ is optimal.

\subsection{Application}

$$
\int_{0}^{t} d t \rightarrow \min \text {. }
$$

subject to the differential constraints

$$
\begin{aligned}
& \dot{x}_{1}=V \cos \theta \\
& \dot{x}_{2}=V \sin \theta+w,
\end{aligned}
$$

The prescribed boundary conditions:

$$
t_{0}=0, x_{10}=0, x_{20}=0 \text {, }
$$

$$
x_{1 f}=1, x_{2 f}=0 .
$$

The constraint of the state are: $\underline{x}_{i} \leq x_{i}(t) \leq \bar{x}_{i}, i=1,2$ with $\underline{x}_{i}=0, \quad \bar{x}_{i}=2$.

This constraint is equivalent to:

$x_{1}(t)-0 \geq 0$,

$x_{2}(t)-0 \geq 0$,

$-x_{1}(t)+2 \geq 0$,

$-x_{2}(t)+2 \geq 0$.

The Hamiltonian is given by:

$H=p_{1}(V \cos \theta)+p_{2}(V \sin \theta+w)-1$.

The Lagrangian is given by:

$L=p_{1}(V \cos \theta)+p_{2}(V \sin \theta+w)-1+\mu_{11}\left(x_{1}(t)-0\right)$

$+\mu_{12}\left(x_{2}(t)-0\right)+\mu_{21}\left(-x_{1}(t)+2\right)+\mu_{22}\left(-x_{2}(t)+2\right)$

where $\mu_{11}, \mu_{12}, \mu_{21}, \mu_{22}$ are Lagrange mutlipliers.

$\dot{p}_{1}=-H_{x 1}=0$

$\dot{p}_{2}=-H_{x 2}=0$,

$0=L_{\theta}=-p_{1} V \sin \theta+p_{2} V \cos \theta$

We show in the following proof that the solution $(x, \theta)$ is an optimal control

Proof We have:

$H_{\theta \theta}=\frac{V^{2}}{V^{2}-w^{2}}>0$.

Then, $H$ is concave in $(x, \theta)$.

$g$ is quasiconcave:

$x_{i}(t)-0 \geq 0, i=1,2$

$\lambda x_{i 1}(t)+(1-\lambda) x_{i 2}(t)-0 \geq \lambda x_{i 1}(t)+(1-\lambda) x_{i 1}(t)=x_{i 1}(t)$

$-x_{i}(t)+2 \geq 0, i=1,2$

$-\lambda x_{i 1}(t)-(1-\lambda) x_{i 2}(t)+2 \geq-\lambda x_{i 1}(t)$

$-(1-\lambda) x_{i 1}(t)+2=x_{i 1}(t)+2$

$\psi$ is quasiconcave: 


$$
\begin{aligned}
& x_{1 f}-1=0 \\
& \lambda x_{1 f 1}(t)+(1-\lambda) x_{1 f 2}(t)-1 \geq \lambda x_{1 f 1}(t) \\
& +(1-\lambda) x_{1 f 1}(t)-1=x_{1 f 1}(t)-1 \\
& x_{2} f-0=0 \\
& \lambda x_{2 f 1}(t)+(1-\lambda) x_{2 f 2}(t)-0 \geq \lambda x_{2 f 1}(t) \\
& +(1-\lambda) x_{2 f 1}(t)-0=x_{2 f 1}(t)
\end{aligned}
$$

then $\psi$ is quasiconcave.

Consequently, $\left(x^{*}, \theta^{*}\right)$ is optimal.

In the constrained case, the numerical experiments ( $\underline{x}_{i}=0, \bar{x}_{i}=2$ ), are summarized below:
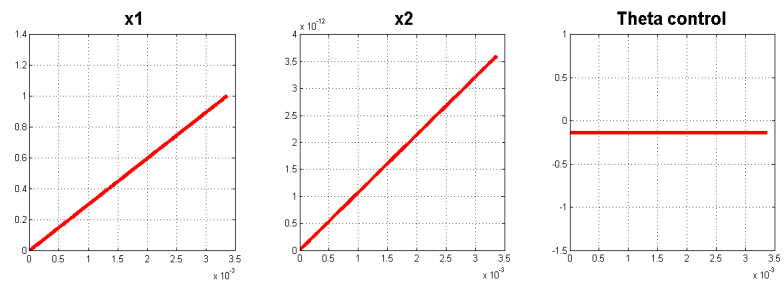

Fig. 7. State and control for a speed of aircraft $V=300$ and of wind $\mathrm{w}=40$ x1

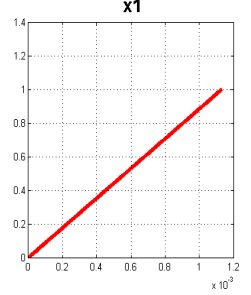

$\mathrm{x} 2$

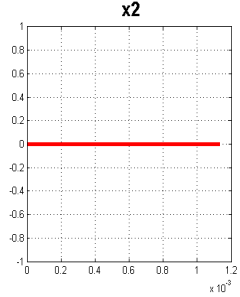

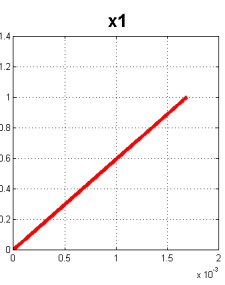
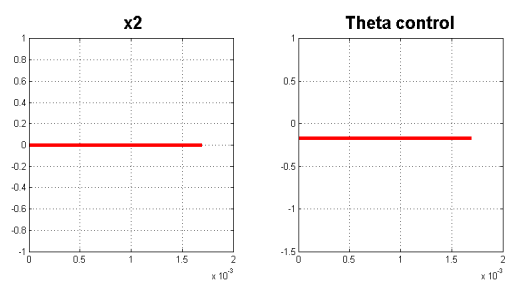

Fig. 10. State and control for a speed of aircraft $V=900$ and of wind $\mathrm{w}=170$

Table 3. Numerical solution

\begin{tabular}{c|c|c|c|c|c}
\hline $\begin{array}{c}V \\
\mathbf{( k m} / \mathbf{h})\end{array}$ & $\begin{array}{c}w \\
\mathbf{k m} / \mathbf{h})\end{array}$ & & $\theta$ & Iterations & $\begin{array}{c}\text { Time } \\
\text { (seconds) }\end{array}$ \\
\hline 300 & 40 & 0.1980 & -0.1980 & 6 & 0.15 \\
350 & 50 & 0.1680 & -0.1433 & 6 & 0.12 \\
400 & 60 & 0.1440 & -0.1506 & 6 & 0.14 \\
450 & 70 & 0.1260 & -0.1562 & 6 & 0.15 \\
500 & 80 & 0.1140 & -0.1607 & 6 & 0.14 \\
600 & 100 & 0.0960 & -0.1674 & 6 & 0.15 \\
700 & 120 & 0.0840 & -0.1723 & 6 & 0.15 \\
800 & 140 & 0.0763 & -0.1759 & 6 & 0.15 \\
900 & 170 & 0.0660 & -0.1900 & 6 & 0.15 \\
\hline
\end{tabular}

Analogously will the case without constraints, we can note that:

- the fast convergence,

- and a very short time of computation.

Then, the numerical procedure is well, adapted to an air-line regulation of aircraft flight.

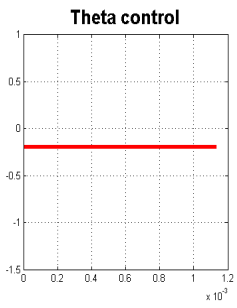

Fig. 8. State and control for a speed of aircraft $V=400$ and of wind $\mathrm{w}=60$ $\mathrm{x1}$

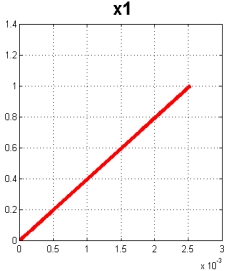

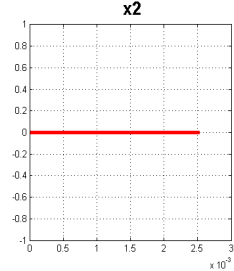

Theta control

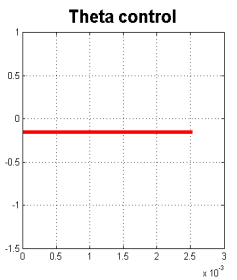

Fig 9. State and control for a speed of aircraft $\mathrm{V}=600$ and of wind $\mathrm{w}=100$

\section{Conclusion}

In this paper, we have solved a problem of optimal control in free final time using Pontryaguin's maximum principle, and for the numerical solution, we used the shooting indirect method to find the transversality conditions in both cases where the state is submitted or not to constraints. We applied this procedure to a navigation problem, where the solution are computed by a numerical way and by an analytical method in the unconstrained case. In this last case, the comparison between the results obtained by the analytical and the numerical methods shows that the solution are similar. We concluded that, in the numerical procedure, the convergence is fast and the computational time are small for both cases where the state is subject or not to constraint.

This is an Open Access article distributed under the terms of the Creative Commons Attribution Licence

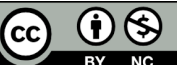

\section{References}

1. Efstathios Bakolas-Panagiotis Tsiotras, Optimal Synthesis of the Zermelo-Markov-Dubins, Problem in a constant Drift Field, J. Optimal theory applications, Springer, 156:469-492. 2013.

2. David G. Hull, Optimal control theory for applications, Springer-Verlag, 2003.

3. J.M.Ortega and W.C.Rheinboldt, Iterative solution of nonlinear equations in several variables. Academic Press, New York, 1970.
4. E.Trelat, Contrôle optimal: théorie et applications, Vuibert, collection Mathématiques Concrètes, 2005.

5. Suresh P.Sethi, Gerald L.Thompsonn. Optimal control theory, applications to management science and economics, second edition, 2000.

6. Walid Bouhafs, Nahla Abdellatif, Frédéric Jean, Jérôme Harmand, Commande optimale en temps minimal d'un procédé biologique 
L. Kahina, P. Spiteri, F. DEMIM, A. Mohamed, A. Nemra and F. Messine/

Journal of Engineering Science and Technology Review 11 (1) (2018) 156-164

d'épuration de l'eau, Arima, Janvier 2013.

7. Alexander J. Zaslavski, Structure of approximate solutions of optimal control problems, Springer, 2013.

8. Leonid D. Akulenko, Problems and Methods of Optimal Control, Springer, 1994.

9. I. H. Mufti, Computational Methods in Optimal Control Problems, Springer, 1970.

10. R. Pytlak, Numerical Methods for Optimal Control Problems with State Constraints, Spinger, 1999.

11. S.Titouche, P.Spiteri, F.Messine, M.Aidene. Optimal control of a large thermic process, Journal of Process Control, Elsevier, Vol. 25 , p. 50-58, janvier 2015.

12. F.Kara, P.Spitéri, F.Messine, M.Aidene. A numerical optimal control method for solving a large thermic process. RAIRO Operations Research, EDP Sciences, Vol. 50 N. 2, p. 297-314, 2016.

13. Y. Park, Morton E. O'Kelly, Fuel burn rates of commercial passenger aircraft: Variations by seat configuration and stage, distance, Journal of Transport Geography, 137-147, 2014.

14. F.Demim, K.Louadj, M.Aidene, A.Nemra, Solution of an Optimal Control Problem with Vector Control using Relaxation Method, Automatic Control and System Engineering Journal, Volume 16, Issue 2, ISSN 1687-4811, ICGST LLC, Delaware, USA, 2016.

15. K.Louadj, P.Spiteri, M.Aidene, F.Messine. An optimal control problem with free final time for aircraft flight with wind (regular paper). Colloque sur l'optimisation et les systèmes d'information (COSI 2014), Bejaia, 08/06/2014-10/06/2014,

16. K.Louadj, P.Spiteri, M.Aidene, F.Messine. Optimal command for the control of the air navigation of an aircraft. The Second International Conference on Electrical Engineering and Control Applications, Constantine, 18/11/2014-20/11/2014.

17. F.Demim, A.Nemra, K.Louadj, M.Hamerlain. Simultaneous localization, mapping, and path planning for unmanned vehicle using optimal control, Advances in Mechanical Engineering, 2018, Vol.10(1),1-25. 